\title{
THE FREE METABELIAN GROUP OF RANK TWO CONTAINS CONTINUOUSLY MANY NONISOMORPHIC SUBGROUPS
}

\author{
G. BAUMSLAG, U. STAMMBACH, AND R. STREBEL
}

(Communicated by Warren J. Wong)

\begin{abstract}
It is proved that the free metabelian group of rank two contains continuously many nonisomorphic subgroups.
\end{abstract}

This short note gives an affirmative answer to the question-attributed to P. Hall - whether a finitely generated metabelian group contains continuously many nonisomorphic subgroups. This question came to the attention of the first author in the early seventies through J. E. Roseblade. To our knowledge it is still open. We resolve this question by proving the following

THEOREM. Given a countable abelian group $A$ there exists a subgroup $U$ of the free metabelian group $G$ of rank two, such that $U_{a b}$ is the direct sum of $A$ and a free abelian group of countable rank.

PrOOF. Let $G$ be the free metabelian group on $\{x, y\}$. We consider the subgroup of $G$ generated by $G^{\prime}$ and $x$. Clearly $x$ generates an infinite cyclic group $C$. Let $L$ denote $G^{\prime}$ as a $Z C$-module, where $C$ acts by conjugation; it is easy to see that $L$ is free of infinite rank. Now let $A$ be any countable abelian group. We view $A$ as a trivial $Z C$-module and write it as quotient of $L$. This gives rise to a short exact sequence

$$
0 \rightarrow K \rightarrow L \rightarrow A \rightarrow 0
$$

of $Z C$-modules. We set $U=\operatorname{gp}(x, K)$. Then

$$
U_{a b}=Z \oplus K /[K, U]=Z \oplus H_{0}(C, K) .
$$

In order to compute $H_{0}(C, K)$ we apply the functor $H_{*}(C,-)$ to the short exact sequence $(\diamond)$. We obtain

$$
\cdots \rightarrow H_{1}(C, L) \rightarrow H_{1}(C, A) \rightarrow H_{0}(C, K) \rightarrow H_{0}(C, L) \rightarrow H_{0}(C, A) \rightarrow 0 .
$$

Since $L$ is a free $Z C$-module, $H_{1}(C, L)=0$ and $H_{0}(C, L)$ is free abelian. Moreover $H_{1}(C, A)=H_{1}(C, Z) \otimes A=A$. It follows that $H_{0}(C, K)$ is a direct sum of $A$ and a free abelian group of countable rank. This proves the theorem.

Department of Mathematics, City College (CUNY), New York, New York 10031 (Current address of G. Baumslag)

Department of Mathematics, ETH, CH 8092, ZuRich, Switzerland (Current address of U. Stammbach and R. Strebel)

Received by the editors January 25, 1988 and, in revised form, May 24, 1988.

1980 Mathematics Subject Classification (1985 Revision). Primary 20F 16, 20E10, 20 J05.

The first author would like to thank the NSF for its support as well as the FIM of ETH for its hospitality and support. 